\title{
Efficiency and harmonic enhancement trends in GaN-based Gunn diodes: Ensemble Monte Carlo analysis
}

C. Sevik, and C. Bulutay

Citation: Appl. Phys. Lett. 85, 3908 (2004); doi: 10.1063/1.1812376

View online: http://dx.doi.org/10.1063/1.1812376

View Table of Contents: http://aip.scitation.org/toc/apl/85/17

Published by the American Institute of Physics

\section{Articles you may be interested in}

Use of $\mathrm{AlGaN}$ in the notch region of GaN Gunn diodes

Applied Physics Letters 95, 143507 (2009); 10.1063/1.3247883

The use of doping spikes in GaN Gunn diodes

Applied Physics Letters 93, 062103 (2008); 10.1063/1.2969779

A comparative investigation on sub-micrometer InN and GaN Gunn diodes working at terahertz frequency Journal of Applied Physics 111, 104514 (2012); 10.1063/1.4721667

Optimized V-shape design of GaN nanodiodes for the generation of Gunn oscillations Applied Physics Letters 104, 073509 (2014); 10.1063/1.4866166

Numerical study of sub-millimeter Gunn oscillations in InP and GaN vertical diodes: Dependence on bias, doping, and length

Journal of Applied Physics 114, 074503 (2013); 10.1063/1.4817884

Searching for THz Gunn oscillations in GaN planar nanodiodes

Journal of Applied Physics 111, 113705 (2012); 10.1063/1.4724350

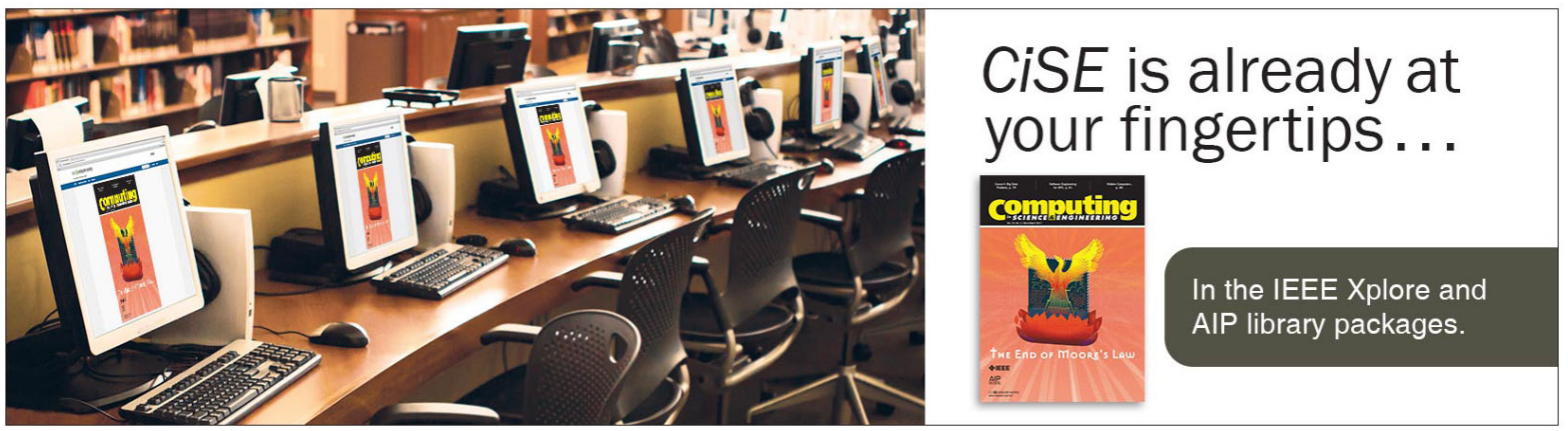




\title{
Efficiency and harmonic enhancement trends in GaN-based Gunn diodes: Ensemble Monte Carlo analysis
}

\author{
C. Sevik ${ }^{\mathrm{a})}$ and C. Bulutay ${ }^{\mathrm{b})}$ \\ Department of Physics, Bilkent University, Bilkent, Ankara 06800, Turkey
}

(Received 9 June 2004; accepted 13 September 2004)

\begin{abstract}
Gallium nitride can offer a high-power alternative for millimeter-wave Gunn oscillators. Hence, an ensemble Monte Carlo-based comprehensive theoretical assessment of efficiency and harmonic enhancement in $n$-type GaN Gunn diodes is undertaken. First, the effects of doping notch/mesa and its position within the active channel are investigated which favors a doping notch positioned next to cathode. It is then observed that the width of the notch can be optimized to enhance the higher-harmonic operation without degrading its performance at the fundamental mode. Next, the effects of dc bias and channel doping density are investigated. Both of these have more significant effects on the higher-harmonic efficiency than the fundamental one. The lattice temperature is observed to have almost no influence up to room temperature but severely degrades the performance above room temperature. As a general behavior, the variations of temperature, channel doping, and the notch width primarily affect the phase angle between the current and voltage wave forms rather than the amplitude of oscillations. Finally, the physical origin of these Gunn oscillations is sought which clearly indicates that the intervalley scattering mechanism is responsible rather than the $\Gamma$ valley nonparabolicity or the effective mass discrepancy between the $\Gamma$ and the lowest satellite valleys. () 2004 American Institute of Physics. [DOI: 10.1063/1.1812376]
\end{abstract}

Gallium nitride with its high negative differential mobility threshold, measured ${ }^{1}$ to be above $200 \mathrm{kV} \mathrm{cm}^{-1}$, is an appealing material for high-power millimeter-wave Gunn diodes. ${ }^{2,3}$ An ever-present objective is to increase the operating frequency of the Gunn diodes, and a promising approach is to operate them at their higher harmonic modes rather than their fundamental mode. Recently, a number of researchers ${ }^{4-8}$ focused on this point addressing the dependence of the higher harmonic efficiency on the doping profile mainly referring to the mature InP technology. The literature on GaN-based Gunn diodes has not yet flourished as the research community up until now mainly focused on the perfection of the material growth quality as well as laser and heterojunction bipolar transistor applications. ${ }^{9}$ Among the recent theoretical reports published on GaN Gunn diodes, Joshi et al. ${ }^{10}$ studied multiple-transit region effects on the output power, $\mathrm{Lü}$ and $\mathrm{CaO}^{11}$ analyzed chaotic dynamics in terahertz generation, and Gružinskis et al. ${ }^{12}$ considered zincblende and wurtzite phases of GaN with an additional $p$-doped barrier region for current limiting.

In this work, our aim is to present a comprehensive theoretical assessment of efficiency and harmonic enhancement in $n$-type GaN Gunn diodes, exploring the effects of doping profile, dc bias, and the lattice temperature. We would like to extract the trends rather than quantitative aspects so that our results can shed light on the physics of Gunn oscillations based on other materials as well. We employ the ensemble Monte Carlo (EMC) technique which has been previously tested in the analysis of InP Gunn diodes. ${ }^{13}$

In a similar work on GaN, Joshi et al. ${ }^{10}$ used the energy of the lowest satellite valley at the $U$ point as $2.2717 \mathrm{eV}$ above the conduction-band minimum, which we also followed in our subsequent work. ${ }^{14}$ As for the current work, we

\footnotetext{
${ }^{a)}$ Electronic mail: sevik@fen.bilkent.edu.tr

${ }^{b)}$ Electronic mail: bulutay@fen.bilkent.edu.tr
}

use more realistic bandstructure data ${ }^{15}$ that place the $U$ valley $1.34 \mathrm{eV}$ above the conduction-band minimum. ${ }^{16}$ All major scattering mechanisms are taken into account, while the scattering tables are formed based on the pseudopotential density of states of GaN. For further details of the parameters, we refer the reader to our previous work. ${ }^{17,18}$

The types of structures we study are shown in Fig. 1. The overall active region length is always kept constant at a value of $\ell_{a}=1.2 \mu \mathrm{m}$. This active region is sandwiched between the heavily doped $n^{+}$regions with $n^{+}=2 \times 10^{18} \mathrm{~cm}^{-3}$; the results were seen to be unaffected when this is increased to $1 \times 10^{19} \mathrm{~cm}^{-3}$. The active region is composed of an active channel with a doping of $n=3 \times 10^{17} \mathrm{~cm}^{-3}$ (unless stated otherwise) and a lightly $n$-doped doping notch having $n^{-}=1$ $\times 10^{16} \mathrm{~cm}^{-3}$ or a doping mesa with $n^{+}=5 \times 10^{17} \mathrm{~cm}^{-3}$.

Our EMC simulations start from a charge-neutral configuration, and unless otherwise stated, are at $300 \mathrm{~K}$. As a standard practice in modeling Gunn diodes (see, Ref. 7 and

(a)

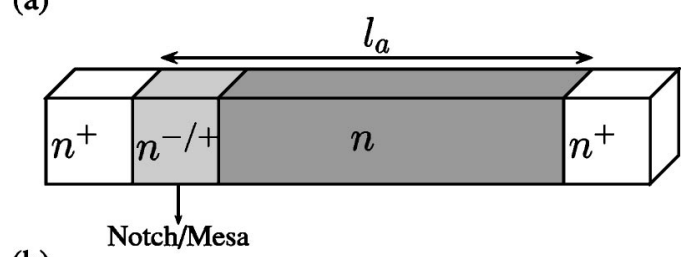

(b)

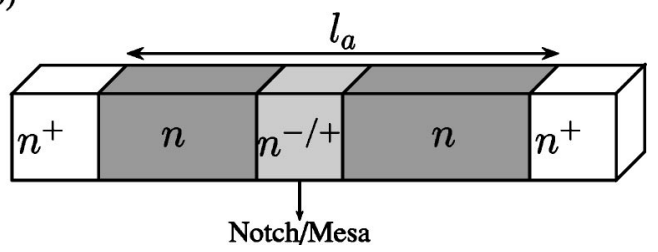

FIG. 1. The types of GaN-based Gunn structures studied: (a) Notch/mesa placed next to cathode and (b) notch/mesa placed in the middle of the active region. 
(a)
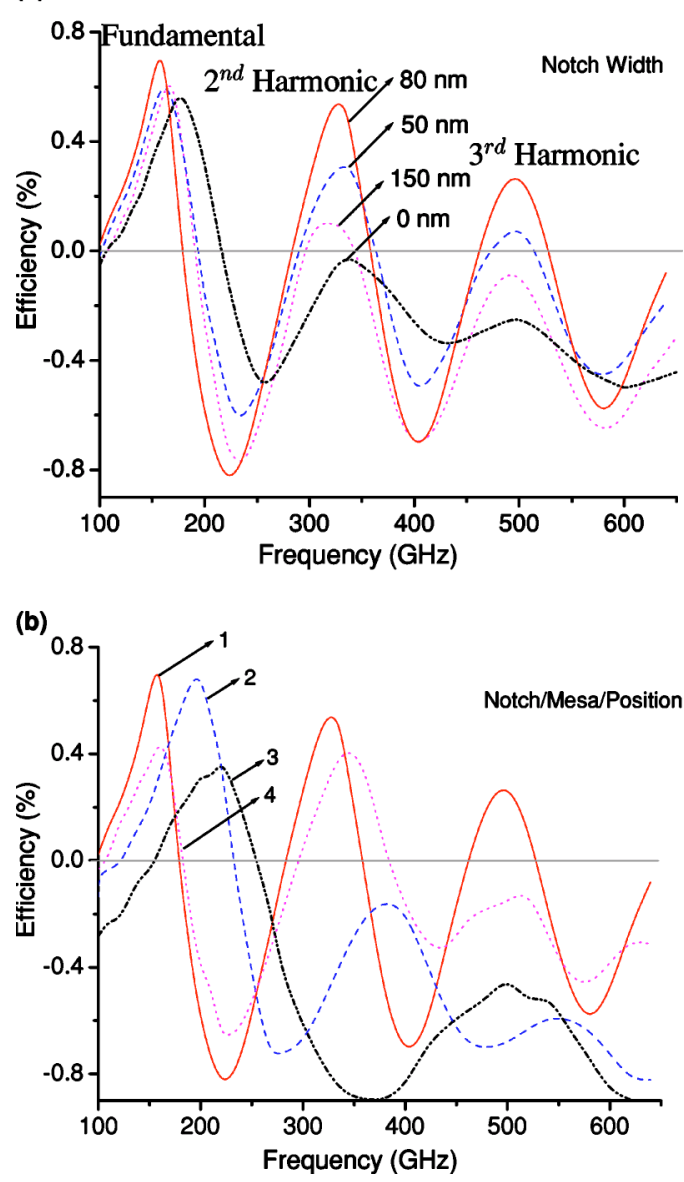

FIG. 2. (Color online) (a) Effect of different doping notch widths. (b) Comparison of the performance of four configuration made from the combinations of notch/mesa placed next to cathode and in the middle of the active region, while keeping the notch $/$ mesa $=80 \mathrm{~nm}$. 1-Notch next to cathode, 2 - mesa next to cathode, 3 - notch in the middle of the active region, and 4 - mesa in the middle of the active region.

references therein), a single-tone sinusoidal voltage of the form $V_{\mathrm{dc}}+V_{\mathrm{ac}} \sin (2 \pi f t)$ is imposed across the structure; if not stated, we use $V_{\mathrm{dc}}=60 \mathrm{~V}$ and $V_{\mathrm{ac}}=15 \mathrm{~V}$. This choice significantly simplifies our frequency performance assessment. Moreover, its validity was checked by relaxing the imposed single-tone sinusoidal voltage across the Gunn diode and connecting it to an external tank circuit with the voltage across the device being self-consistently updated at each simulation step through solving the Gunn diode and the external circuit. ${ }^{14}$ For both cases, the phase relations were found to be very similar. ${ }^{14}$ To assure that both amplitude and phase have stabilized, simulations are lasted for at least 0.2 ns. The current wave form is sampled at every $80 \mathrm{fs}$ while the simulation time step is $0.4 \mathrm{fs}$.

The oscillator efficiency, is defined as $\eta=p_{\mathrm{ac}} / P_{\mathrm{dc}}$, where $P_{\mathrm{ac}}$ is the time-average generated ac power and $P_{\mathrm{dc}}$ is the dissipated dc power by the Gunn diode. First, the effect of the doping notch width [positioned next to cathode, c.f. Fig. 1(a)] is investigated. It is observed that there is an optimum notch width, for our case, around a value of $80 \mathrm{~nm}$, at which the second-harmonic efficiency approaches that of the fundamental mode value as seen in Fig. 2(a). Here, the fundamental mode corresponds to the frequency $v_{d \text {,sat }} / \ell_{a}$ for a given active region length $\ell_{a}$, where $v_{d \text {, sat }}$ is the saturation drift velocity; in the $n$th harmonic mode there exist $n$ Gunn domains traveling down the channel at each instant. ${ }^{14}$ However,
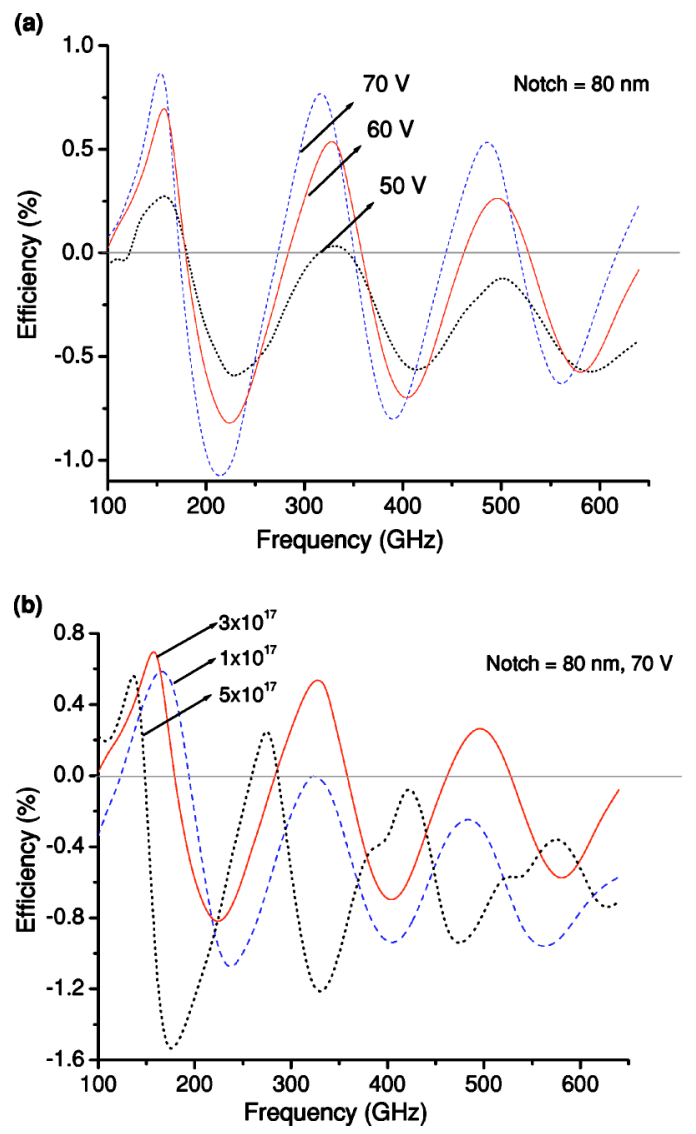

FIG. 3. (Color online) (a) rf conversion efficiencies vs frequency for several dc bias voltages with the rf amplitude being scaled accordingly; $80 \mathrm{~nm}$ notch device. (b) rf conversion efficiency vs frequency for channel dopings; $80 \mathrm{~nm}$-notch device at $70 \mathrm{~V} \mathrm{dc}$ bias is used.

we would like to point out that the value of the optimum notch width is sensitive to the material parameters and our previous analysis with the bandstructure data of Ref. 10 resulted in a wider width. ${ }^{14}$ On the other hand, a doping mesa rather than a notch was suggested to enhance the harmonic content of the current wave form. ${ }^{4,6}$ To elucidate this point, Fig. 2(b) compares the performance of four configurations made from the combinations of notch/mesa placed next to cathode and in the middle of the active region. It is seen that notch placed next to cathode (labeled as 1) performs the best among all. A source of curiosity regarding Fig. 2(b) is the substantial frequency shift for the notch placed in the middle (labeled as 3) and mesa placed next to the cathode (labeled as 2). A detailed time-domain investigation of these structures shows that for both cases almost half of the active region is inactive in the domain nucleation processes, hence remarkably decreasing the transit time of the Gunn domains.

In Fig. 3, the effects of dc bias and channel doping are displayed. While changing the applied dc voltage, we need to scale the rf amplitude accordingly not to lose the grounds for efficiency comparison. The applied dc voltage has to be above a critical value so that the device is biased in the negative differential mobility regime. Therefore, increasing bias has favorable effect on the fundamental and harmonic efficiencies. The channel doping on the other hand is relatively less influential on the fundamental mode efficiency than the higher harmonic modes, so that for a given notch width and bias voltage, there is an optimum channel doping that favors the harmonic enhancement. However, there is a 

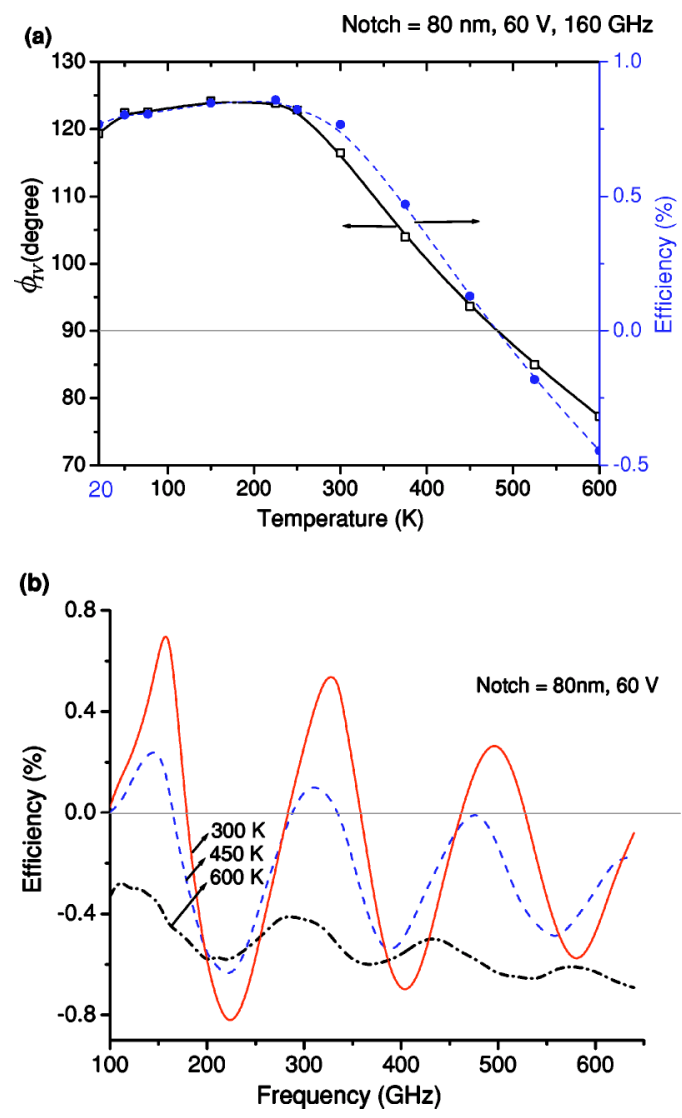

FIG. 4. (Color online) (a) Temperature vs phase angle, $\phi_{\mathrm{IV}}$, between the current and voltage wave forms, and efficiency; $80 \mathrm{~nm}$-notch device at $60 \mathrm{~V}$ bias and its fundamental frequency $(160 \mathrm{GHz})$ is used. (b) rf conversion efficiency and frequency for several lattice temperatures; $80 \mathrm{~nm}$-notch device at $60 \mathrm{~V}$ bias is used.

lower threshold for the channel doping as it determines the dielectric domain nucleation time that needs to be much shorter than the domain transit time through the active region. ${ }^{19}$

For high power Gunn diodes, thermal heating can be an important issue. At the expense of neglecting thermal gradient effects, we simply consider the uniform lattice temperature effects on the efficiency. As can be observed in Fig. 4(a), up to room temperature, there is no sensible variation, whereas above the room temperature performance increasingly degrades becoming passive above $480 \mathrm{~K}$ in the case considered. The source of this degradation is not due to a reduction in the oscillation amplitudes but rather due to phase angle between the current and voltage wave forms shifting away from antiphase $\left(180^{\circ}\right)$ to orthogonal $\left(90^{\circ}\right)$ and finally towards dissipative $\left(0^{\circ}\right)$ while the temperature increases. As a matter of fact, the phase angle difference is always far below $180^{\circ}$ due to the finite capacitance of the Gunn diode which is not cancelled by an external inductor under the imposed single-tone sinusoidal bias condition that is employed in this work. Figure 4(b) further illustrates that the increase in the lattice temperature does not significantly shift the frequency but diminishes the efficiency globally. Therefore, good heat sinking is required in these diodes.

On the physical origin of the Gunn oscillations, there exists a number of possible sources, such as the $\Gamma$ valley nonparabolicity, intervalley transfer mechanism to satellite valleys, and, as recently suggested, ${ }^{11}$ ballistic transport through the $\Gamma$ valley band inflection point when located below the lowest satellite valley energy. In our EMC treatment, which is essentially based on spherical bands hence without any band inflection points, this last choice cannot play a role. To identify the effect of $\Gamma$ valley nonparabolicity, switching to a parabolic band further increases the rf generation efficiency whereas increasing the nonparabolicity decreases it. This rules out the fact that it can be the cause, and one can reason it as the nonparabolicity delaying the electrons under the electric field's acceleration from reaching the satellite valley energy. On the effective mass discrepancy, the lowest satellite $U$ valley is more than twice as heavy as the $\Gamma$ valley ${ }^{15}$ which advocates the transfer from a light to a heavy band as an additional driving mechanism. Interestingly, equating these two masses decreases the efficiency somewhat but it does not quench it. As a matter of fact, we identified the optical deformation potential field which leads to intervalley scattering as the most important parameter that controls the efficiency. Fortunately, its value for $\mathrm{GaN}$ has recently been rigorously determined by Akis et al. ${ }^{20}$

In summary, we have analyzed the trends in efficiency and harmonic enhancement in GaN Gunn diodes under doping profile, bias, and temperature variations. The key parameter in all these efficiency considerations has been the phase angle difference between the current and voltage waveforms. Our extensive simulations indicate that the carrier dynamics in GaN can be tailored by an optimum choice of doping profile, temperature, and bias conditions so that the efficiency of higher harmonic Gunn oscillations can be boosted.

This work was supported by The Scientific and Technical Research Council of Turkey (COST 288 Action). The authors thank B. Tanatar for his continuous encouragement.

${ }^{1}$ M. Wraback, H. Shen, J. C. Carrano, T. Li, J. C. Campbell, M. J. Schurman, and I. T. Ferguson, Appl. Phys. Lett. 76, 1155 (2000).

${ }^{2}$ E. Alekseev, D. Pavlidis, N. X. Nguyen, and D. E. Grider, IEEE Trans. Microwave Theory Tech. 48, 1694 (2000).

${ }^{3}$ E. Alekseev and D. Pavlidis, Solid-State Electron. 44, 941 (2000).

${ }^{4}$ S. H. Jones, G. B. Tait, and M. Shur, Microwave Opt. Technol. Lett. 5, 354 (1992).

${ }^{5}$ A. I. Mikhaŭlov and D. M. Lerner, Tech. Phys. Lett. 24, 836 (1998).

${ }^{6}$ R. Judaschke, IEEE Trans. Microwave Theory Tech. 48, 719 (2000).

${ }^{7}$ G. M. Dunn and M. J. Kearney, Semicond. Sci. Technol. 18, 794 (2003).

${ }^{8}$ P. Shiktorov, E. Starikov, V. Gružinskis, S. Pérez, T. González, L. Reggiani, L. Varani, and J. C. Vaissière, IEEE Trans. Electron Devices, 50, 1171 (2003).

${ }^{9}$ O. Ambacher, J. Phys. D 31, 2653 (1998).

${ }^{10}$ R. P. Joshi, S. Viswanadha, P. Shah, and R. D. del Rosario, Appl. Phys. 93, 4836 (2003).

${ }^{11}$ J. T. Lü, and J. C. Cao, Semicond. Sci. Technol. 19, 451 (2004).

${ }^{12}$ V. Gružinskis, P. Shiktorov, E. Starikov, and J. H. Zhao, Semicond. Sci. Technol. 16, 798 (2001).

${ }^{13}$ V. Gružinskis, E. Starikov, P. Shiktorov, L. Reggiani, and L. Varani, J. Appl. Phys. 76, 5260 (1994).

${ }^{14}$ C. Sevik and C. Bulutay, Semicond. Sci. Technol. 19, 188 (2004).

${ }^{15}$ C. Bulutay, B. K. Ridley, and N. A. Zakhleniuk, Phys. Rev. B 62, 15754 (2000).

${ }^{16}$ C. -K. Sun, Y. -L Huang, S. Keller, U. K. Mishra, and S. P. DenBaars, Phys. Rev. B 59, 13535 (1999).

${ }^{17}$ C. Bulutay, Semicond. Sci. Technol. 17, L59 (2002).

${ }^{18}$ C. Sevik and C. Bulutay, Appl. Phys. Lett. 83, 1382 (2003).

${ }^{19}$ M. Shur, Introduction to Electronic Devices (Wiley, New York, 1996).

${ }^{20}$ R. Akis, M. Dür, and S. M. Goodnick, Physica B 314, 42 (2002). 